\title{
A generalized entropy measuring quantum localization
}

\author{
B. Mirbach ${ }^{\dagger}$ and H. J. Korsch* \\ ${ }^{\dagger}$ MPI für Physik komplexer Systeme, D-01187 Dresden, Germany, \\ e-mail: mirbach@mpipks-dresden.mpg.de \\ ${ }^{*}$ FB Physik, Universität Kaiserslautern, D-67653 Kaiserslautern, Germany, \\ e-mail: korsch@physik.uni-kl.de
}

July 4, 1997

\begin{abstract}
We present an entropy concept measuring quantum localization in dynamical systems based on time averaged probability densities. The suggested entropy concept is a generalization of a recently introduced [PRL 75, 326 (1995)] phase-space entropy to any representation chosen according to the system and the physical question under consideration. In this paper we inspect the main characteristics of the entropy and the relation to other measures of localization. In particular the classical correspondence is discussed and the statistical properties are evaluated within the framework of random vector theory. In this way we show that the suggested entropy is a suitable method to detect quantum localization phenomena in dynamical systems.
\end{abstract}




\section{Introduction}

The ultimate goal in the rapidly developing field of Quantum Chaos is to obtain fingerprints of classical chaos in quantum systems. Considerable progress has been made both in semiclassical quantization of classical chaotic systems as well as in the statistical description of quantum spectra within the framework of random matrix theory (see, e.g., $[1,2,3]$ ). Another approach towards an understanding of the role of chaos in quantum mechanics is to analyze the localization properties of quantum states. In several numerical studies, it has been demonstrated that quantum states which are completely delocalized on a classically ergodic region in phase space (a 'chaotic sea') can be described within the context of random vector theory $[4,5,6,7]$. Several mechanisms, however, lead to limitations of quantum ergodicity for a finite value of $\hbar$ :

(i) Dynamical localization: This so-called quantum suppression of classical diffusion has been comprehensively investigated for the kicked rotor and has been explained in analogy to the Anderson localization in disordered solids $[8,9]$. Moreover, dynamical localization seems to be a universal phenomenon, related to the time scale (the so-called break-time or Heisenberg time) on which quantum time evolution saturates due to the discreteness of the spectrum (see, e.g., [10]).

(ii) Barrier action of tori and cantori: Localization on quantized invariant tori is well described within the semiclassical EBK-theory. This theory, originally developed for integrable systems, proved to be also applicable to KAM-tori in systems with mixed phase-space if the corresponding stability regions are large enough compared to $h^{f}$ (for examples see $[11,12]$ ). The numerical work by Geisel et al. [13] showed that the, so-called, cantori can also act as barriers like tori. A general (semiclassical) theory for this phenomenon is, however, still missing, at least to the knowledge of the authors.

Besides these 'stronger types' of localization, which are characterized by an exponential decay outside of a certain region, also weaker forms of localization have been encountered as there are 'scars', localization on 'ghost orbits' and on regular phase-space structures ('islands') much smaller than Planck's constant. These types of localization may, however, not be intermixed with the so-called weak localization. This term denotes the quantum enhancement in the autocorrelation function of a wave packet due to constructive interference of time-reversed paths.

Quantitative studies of these localization phenomena require an appropri- 
ate measure for the degree of localization. A general problem faced in studying, e.g., the localization of eigenstates are inherent quantum fluctuations which do not vanish in the classical limit and hence cause large fluctuations and deviations in the measures of localization from the corresponding classical values. It has therefore been difficult to perform quantitative studies of the weaker forms of localization which yield statistical significant results.

In Sect. 2, we briefly review some measures of localization commonly used and dicuss their statistical properties within the framework of random vector theory. In Sect. 4, an entropy concept based on time-averaged quantum densities is introduced, which is a generalization of the phase-space entropy first introduced in [14]. Here we evaluate the statistical properties of the introduced entropy, in particular the scaling with $\hbar$ and the classical limit and compare it with the traditionally used measures reviewed in Sect. 2.

\section{Measures of localization}

We assume for convenience that $\{|n\rangle\}_{n}$ is a complete set of normalized eigenstates of the Hamiltonian $\hat{H}$, numbered by a (multi-)index $n$, i.e. we assume the system to be bound. Thus one has the decomposition of unity

$$
\mathbb{I}=\sum_{n}|n\rangle\langle n|,
$$

on the underlying Hilbert space. On the other hand, we assume to have some other set $\{|\alpha\rangle\}_{\alpha}$ of states also forming a basis, which might, however, be overcomplete like, e.g., coherent states. Also the representation in coordinate or momentum space can be treated within this context. The corresponding decomposition of unity reads therefore in general

$$
\mathbb{I}=\int \mathrm{d} \mu_{\alpha}|\alpha\rangle\langle\alpha|
$$

The choice of a basis $\{|\alpha\rangle\}$ depends on the system and the physical problem under investigation. In perturbed systems, the eigenstates of the unperturbed Hamiltonian may provide some 'natural basis'. For periodically kicked or continuously driven rotor systems one usually computes eigenstates in an expansion of free-rotor states, which allow for a definition of a localization length in momentum space (see. e.g. $[10,15]$ ). In other systems, one may 
be interested in phase-space localization giving rise to the introduction of coherent states. An appropriate measure for the degree of delocalization of a state $|\psi\rangle$ in the basis $\{|\alpha\rangle\}$ is the Shannon entropy

$$
S_{\{\alpha\}}=-\int \mathrm{d} \mu_{\alpha} p_{\alpha} \ln p_{\alpha},
$$

with $p_{\alpha}=|\langle\psi \mid \alpha\rangle|^{2}$ being the die projections of a unit vector $|\psi\rangle$ onto the basis states. An alternative measure is the, so-called, mean inverse participation ratio

$$
\xi_{\{\alpha\}}^{-1}=\int \mathrm{d} \mu_{\alpha} p_{\alpha}^{2},
$$

(see, e.g., $[16,17])$. These different measures of localization can, however, be subsumed under the concept of a Renyi entropy

$$
S_{\{\alpha\}}^{(\gamma)}=\frac{1}{1-\gamma} \ln \int_{\alpha} \mathrm{d} \mu_{\alpha} p_{\alpha}^{\gamma}
$$

which is defined for $0<\gamma<\infty, \gamma \neq 1$ (see, e.g., [18]). The comparison with (4) yields immediately $S^{(2)}=\ln \xi_{\{\alpha\}}$ and the Shannon entropy (3) results from the Renyi entropy in the limit $\gamma \searrow 1$. Between the different Renyi entropies there is the inequality

$$
S^{(\gamma)} \geq S^{\left(\gamma^{\prime}\right)} \text { for } \quad \gamma \leq \gamma^{\prime}
$$

In the following we will concentrate on the Shannon entropy (3) which can be interpreted as the missing information about the state $|\psi\rangle$ when only its projections $p_{\alpha}$ are known. A comparison with the information entropy (or von Neumann entropy) $S_{\mathrm{v} . \mathrm{N}}=-\operatorname{tr}(\hat{\varrho} \ln \varrho$ ) of a density matrix $\hat{\varrho}$ shows that the entropy (3) of a pure state $|\psi\rangle$ corresponds to the information entropy of a density matrix

$$
\hat{\varrho}=\int \mathrm{d} \mu_{\alpha} p_{\alpha}|\alpha\rangle\langle\alpha|
$$

which results from the projector onto $|\psi\rangle$ by cancelling the off-diagonal elements in the $\{\alpha\}$-representation. In contrast to the information entropy, the Shannon entropy (3) is basis-dependent. For the particular case of harmonic oscillator coherent states as a basis, i.e. $\{|\alpha\rangle=|p, q\rangle\}$, the corresponding 
entropy was introduced by Wehrl [19]. A discussion of the properties of the Wehrl entropy

$$
S_{W}=-\int \frac{\mathrm{d}^{f} p \mathrm{~d}^{f} q}{(2 \pi \hbar)^{f}}|\langle p, q \mid \psi\rangle|^{2} \ln |\langle p, q \mid \psi\rangle|^{2}
$$

and the relation to the uncertainty principle can be found in [20]. Within an operational approach, the Wehrl entropy can be interpreted as a sampling entropy with coherent states as a filter. A comprehensive presentation of this approach was recently given by Bužek et al. [21, 22].

Independent of the chosen basis $\{|\alpha\rangle\}$ one has the inequality

$$
S_{\{\alpha\}} \geq S_{\text {v.N. }}
$$

which can be easily verified using the concavity of the function $f(x)=x \ln x$. For pure states, this inequality is obviously trivial, because $S_{\mathrm{v} . \mathrm{N}}=0$.

In several studies the entropy (3) has been used to measure the degree of localization of eigenstates in a certain 'natural basis', also in order to separate the states into 'regular' and 'chaotic' ones my measuring their degree of localization (for recent applications see [23, 24]). This distinction is, however, limited on the one hand by weaker localization phenomena as mentioned above and on the other hand by strong fluctuations in the entropy of 'chaotic states'. These fluctuations limit also the accuracy with which a localization length can be determined and often some kind of averaging has to be done.

The fluctuations in the entropy result from inherent quantum fluctuations in the projections $p_{\alpha}$, which can be described within the context of random vector theory. A first comprehensive discussion of the entropy of random vectors was presented by Wooters [25] and Życzkowski [26]. Here we give only a brief summary of their results as far as necessary for the following. But we will present a novel straightforward derivation of the expression for the mean entropy and in addition also for the mean fluctuations of the entropy.

\section{The entropy of random vectors}

The basic idea of random vector theory is to assign to each unit vector in the underlying Hilbert space the same probability, e.g., to be an eigenstate of a certain operator. Formally this means to define a probability density 
on Hilbert space which is invariant under the group of unitary transformation of the system. Assuming the Hilbert space to be $N$-dimensional and $\{|j\rangle, j=1, \ldots, N\}$ to be an orthogonal basis, the probability distribution on the corresponding space $K=\left\{c_{1}, \ldots, c_{N}\right\}$ of coefficients $c_{j}=\langle j \mid \psi\rangle$ has the form

$$
\varrho\left(c_{1}, \ldots, c_{N}\right)=\text { const } \times \delta\left(1-\sum_{j=1}^{N}\left|c_{j}\right|^{2}\right) .
$$

From this density, the probability distribution $P\left(\left|c_{j}\right|^{2}=p\right)$ for the projection on any given vector can be derived (see, e.g., [27]). The result is

$$
P_{\nu}(p)=\frac{\Gamma(N \nu / 2)}{\Gamma(\nu / 2) \Gamma((N-1) \nu) / 2)} p^{\nu / 2-1}(1-p)^{(N-1) \nu / 2-1} .
$$

Due to the isotropy of the density (10), the probability distribution (11) is independent of the direction of projection. The parameter $\nu=1,2,4$ distinguishes the three universality classes (orthogonal, unitary, symplectic) of the system [28].

For large $N$ the distribution approaches a $\chi^{2}$-distribution

$$
P_{\nu}(p) \approx N \chi_{\nu}^{2}(N p)=\left(\frac{\nu N}{2}\right)^{\nu / 2} \frac{1}{\Gamma(\nu / 2)} p^{(\nu / 2)-1} \exp \left(-\frac{\nu N}{2} p\right) .
$$

In this limit, the fluctuations of the projections onto different unit vectors become independent of each other, i.e. the correlation of different projections due to the normalization of the state $|\psi\rangle$ vanishes since $\bar{p}=1 / N \rightarrow 0$ for $N \rightarrow 0$. The mean product of the projections onto two unit vectors $|\alpha\rangle$ and $\left|\alpha^{\prime}\right\rangle$ can easily be derived to be

$$
\overline{p_{\alpha} p_{\alpha^{\prime}}}=\frac{\nu+2\left|\left\langle\alpha \mid \alpha^{\prime}\right\rangle\right|^{2}}{N(N \nu+2)}
$$

An important point is that the fluctuations of the projections fulfill the relation

$$
(\Delta p)^{2}=\frac{2}{\nu} \bar{p}^{2}
$$

for large $N$. This means that the relative fluctuations are of order 1 and independent of $N$, i.e. they do not vanish in the classical limit $N \rightarrow \infty$. 
In several studies it was shown that (in the absence of dynamical localization) the fluctuations of eigenstate components in some chosen natural basis are in accordance with the predicted distribution function $(12)[4,5,6,7]$. This result can be interpreted by saying that the eigenbasis and the chosen 'natural basis' contain no information of each other, i.e. they appear to be random to each other. If, e.g., the underlying 'natural basis' is the basis of an unperturbed Hamiltonian, this result may be interpreted as the quantum analogue of the classical destruction of invariant tori by a (strong) perturbation. In this case, the invariant phase-space structure does not contain any more tori of the unperturbed system. If, however, the perturbed system is still (almost) integrable, the phase-space of the system is still stratified by invariant tori which originate from those of the unperturbed system by smooth deformation. In this case, the quantum states - since they are supported by quantized invariant tori - do still contain information about the unperturbed states and their expansion coefficients will show a non universal distribution.

Using the distribution function (11) one can compute the average entropy of a random vector:

$$
\bar{S}_{\nu}=-N \int \mathrm{d} p P_{\nu}(p) p \ln p
$$

Jones [29] showed by an involved analysis that the integral (15) allows for an analytical evaluation with the result

$$
\bar{S}_{\nu}=\Psi(\nu N / 2+1)-\Psi(\nu / 2+1),
$$

where $\Psi(x)=\Gamma^{\prime}(x) / \Gamma(x)$ is the digamma function [30]. For the case of a Gaussian distribution (i.e. $\nu=1$ ) this exact result was already given by Izrailev [31]. There is, however, a much simpler straight forward derivation of (16) by using Euler's beta function [32]:

$$
B(w, z)=\int_{0}^{1} \mathrm{~d} p p^{w-1}(1-p)^{z-1}=\frac{\Gamma(w) \Gamma(z)}{\Gamma(w+z)} .
$$

Rewriting the integral (15) as

$$
\bar{S}_{\nu}=-\frac{\partial}{\partial w} \ln B(w, z)
$$


with parameters $w=\nu / 2+1$ and $z=(N-1) \nu / 2$ immediately leads to (16).

Using the asymptotic series of the digamma-function $\Psi(x)=\ln (x)+$ $\mathcal{O}(1 / x)$ one obtains for large $N$ the asymptotic behavior of the mean entropy:

$$
\begin{aligned}
\bar{S}_{\nu} & =\ln N-c_{\nu}, \\
c_{\nu} & =\ln \nu / 2-\Psi(\nu / 2+1),
\end{aligned}
$$

which follows also from evaluating the integral (15) within the $\chi^{2}$-approximation (12) $[25,26]$. This result states that the average entropy of a 'random' unit $N$-vector is the maximum entropy, $\ln N$, minus a ( $N$-independent) constant $c_{\nu}$, which is determined by the symmetry class of the system only. The origin of the reduction of the mean entropy compared to the maximum entropy are the non-vanishing relative fluctuations in the vector components. Casati et al. [10] realized that these deviations have to be taken into account when defining an appropriate number of occupied states or a localization length on basis of this entropy.

For deriving the standard deviation of the entropy, one has to start from the joint probability density (10) in order to include also the influence of the correlations between different vector components. Therefore this calculation is, though elementary, very lengthy such that we omit here the derivation. The result is:

$$
(\Delta S)^{2}=\frac{2}{\nu N+2}\left[\left(\frac{\nu}{2}+1\right) \Psi^{\prime}\left(\frac{\nu}{2}+1\right)-\left(\frac{N \nu}{2}+1\right) \Psi^{\prime}\left(\frac{N \nu}{2}+1\right)\right],
$$

where $\Psi^{\prime}$ denotes the derivative of the digamma function. Inserting the asymptotic expansion of the the digamma function, one obtains for large $N$ the approximation

$$
(\Delta S)^{2}=\frac{2}{\nu N+2}\left[\left(\frac{\nu}{2}+1\right) \Psi^{\prime}\left(\frac{\nu}{2}+1\right)-1\right]
$$

The derivatives of the digamma functions are intimately related to the zeta function; the values of $\Psi^{\prime}\left(\frac{\nu}{2}+1\right)$ can can be obtained recursively from $\zeta(2)$ 
[30]. The result for the three universality classes is explicitely:

$$
(\Delta S)^{2}=\frac{1}{N}\left\{\begin{array}{ll}
\frac{3 \pi^{2}}{2}-14 & \nu=1 \\
\frac{\pi^{2}}{3}-3 & \nu=2 \\
\frac{\pi^{2}}{4}-\frac{19}{8} & \nu=4
\end{array} .\right.
$$

This asymptotic result has already been presented by Wooters for the case $\nu=2[25]$. Of interest is the scaling of $\Delta S$ with $1 / \sqrt{N}$; the fluctuations of the mean inverse participation ratio are of the same order of magnitude.

The formulas $(19,23)$ explain the behavior of the entropy of 'chaotic eigenstates' computed in some natural basis, as, e.g., done in [23, 24] to separate 'chaotic' from 'regular' quantum states. The small but not negligible fluctuations in the individual entropies make it, however, difficult to distinguish states which show some weaker form of localization in a statistical significant way.

Rather than computing the entropy $S_{\{\alpha\}}(n)=-\int \mathrm{d} \mu_{\alpha} p_{n, \alpha} \ln p_{n, \alpha}$ of the eigenstates $\{|n\rangle\}$ of a full Hamiltonian in some natural basis $\{|\alpha\rangle\}$, one may instead compute the entropy

$$
S_{\{n\}}(\alpha)=-\sum_{n} p_{n, \alpha} \ln p_{n, \alpha}
$$

of the chosen basis vectors in the eigenbasis of the system. When using coherent states as a basis $\{|\alpha\rangle\}$, the map $\alpha \mapsto S_{\{n\}}(\alpha)$ defines a quantum phase-space mapping showing local localization phenomena. Such a plot was suggested by Życzkowski [26].

One can easily verify that the Shannon entropy of any vector $|\alpha\rangle$ in the eigenbasis of a Hamiltonian corresponds to the information entropy $S_{\{n\}}(\alpha)=$ $-\operatorname{tr}\left(\bar{\varrho}_{\alpha} \ln \bar{\varrho}_{\alpha}\right)$ of the time averaged projector

$$
\bar{\varrho}_{\alpha}=\lim _{T \rightarrow \infty} \frac{1}{T} \int_{0}^{T}|\alpha(t)\rangle\langle\alpha(t)| \mathrm{d} t
$$

provided that the eigenstates are non-degenerate. This measure of localization was suggested by Thiele and Stone [33] as a dynamical approach to quantum chaos. It has, however, the disadvantage that there is no direct classical counterpart to this entropy and that it is dominated by non-negligible quantum fluctuations of order $1 / \sqrt{N}$ as described above. 


\section{Entropy of time averaged probability den- sities}

Here we shall suggest an entropy concept based on time averaged probability densities which has a direct classical counterpart. In addition, it will be shown that the relative fluctuations of this entropy due to inherent quantum fluctuations are reduced to an order of $1 / N$ thus being small in the semiclassical regime compared to, e.g., the fluctuations in (24) of order $1 / \sqrt{N}$.

The basic quantity we consider are the time averaged probability densities obtained for the time evolved basis states $|\alpha\rangle$ :

$$
\varrho\left(\alpha, \alpha^{\prime}\right)=\lim _{t \rightarrow \infty} \frac{1}{T} \int_{0}^{T} \mathrm{~d} t\left|\left\langle\alpha(t) \mid \alpha^{\prime}\right\rangle\right|^{2}
$$

This quantity has already been considered long time ago by Nordholm and Rice to quantify the vibrational relaxation in isolated molecules [34]. It describes the statistical relaxation of the propagation of the states $|\alpha\rangle$ in the sense that the time-dependent projections $\varrho\left(\alpha, \alpha^{\prime}, t\right)=\left|\left\langle\alpha(t) \mid \alpha^{\prime}\right\rangle\right|^{2}$ will fluctuate around these mean values for all times.

Expanding the basis vectors $|\alpha\rangle$ in the eigenbasis $\{|n\rangle\}$ of the Hamiltonian, one obtains

$$
\varrho\left(\alpha, \alpha^{\prime}\right)=\sum_{n}|\langle\alpha \mid n\rangle|^{2}\left|\left\langle\alpha^{\prime} \mid n\right\rangle\right|^{2}=\sum_{n} p_{\alpha, n} p_{\alpha, n^{\prime}}
$$

provided the the eigenbasis is non-degenerat. From (27) one recognizes immediately that the probability matrix $\varrho\left(\alpha, \alpha^{\prime}\right)$ is symmetric. The diagonal

elements $\varrho(\alpha, \alpha)$ are the mean inverse participation ratios $\xi_{\{n\}}^{-1}(\alpha)=\sum_{n} p_{\alpha, n}^{2}$ of the state $\alpha$ in the Hamiltonian eigenbasis. Rather than using onle these diagonal elements as a measure for localization, we suggest to compute the entropy from the probability distributions given by the columns (or rows) of the matrix, i.e.

$$
S(\alpha)=-\int \mathrm{d} \mu_{\alpha^{\prime}} \varrho\left(\alpha, \alpha^{\prime}\right) \ln \varrho\left(\alpha, \alpha^{\prime}\right)
$$

This entropy has two important properties which quantify it as a better quantity offering a dynamical approach towards measuring quantum localization in dynamical systems. 


\section{The classical entropy}

The probability distribution $\varrho\left(\alpha, \alpha^{\prime}\right)$ has a classical counterpart, which can be defined in a entirely analogous manner on basis of time-averaged probability densities on phase-space. Consider first a discrete division of classical phasespace in cells of size $\hbar^{f}$. Then one defines $\varrho(i, j)$ to be the time averaged probability for a trajectory initially located in cell $i$ to visit cell $j$. The entropy

$$
S^{\mathrm{cl}}(i)=-\sum_{j} \varrho^{\mathrm{cl}}(i, j) \ln \varrho^{\mathrm{cl}}(i, j)
$$

of these classical discrete probability distributions was introduced by Núñez et al. [35] as an indicator of chaos in models of celestial dynamics. An interesting property of the classical entropy is its scaling with the box size, which is per definition the information dimension of the invariant set cell $i$ belongs to, i.e.

$$
D=\lim _{\hbar_{0} \rightarrow 0} \frac{S(i)}{\ln \hbar_{0}^{-1 / 2}},
$$

where $\hbar_{0}$ is a dimensionless scaling parameter proportional to $\hbar$. This dimension may also be fractal.

Alternatively to a discrete division of phase-space a continuous partition using distribution functions $w_{\alpha}^{\mathrm{cl}}(p, q)$ with a scaling parameter $\hbar$ can be defined. By propagating each of these distributions by Liouville's equation and taking the overlap with the initial distribution functions, i.e.

$$
\varrho^{\mathrm{cl}}\left(\alpha, \alpha^{\prime}, t\right)=\int \mathrm{d} p \mathrm{~d} q w_{\alpha}^{\mathrm{cl}}(p, q, t) w_{\alpha^{\prime}}^{\mathrm{cl}}(p, q),
$$

one obtains after time averaging the classical probability distribution

$$
\varrho^{\mathrm{cl}}\left(\alpha, \alpha^{\prime}\right)=\lim _{T \rightarrow \infty} \frac{1}{T} \int_{0}^{T} \mathrm{~d} t \varrho^{\mathrm{cl}}\left(\alpha, \alpha^{\prime}, t\right)
$$

in analogy to the quantum density (26). In case that the classical dynamics

is mixing, the last step of time averaging is, however, non essential, since then the time-dependent distribution (31) converges by definition. 
The classical probability distribution (32) defined in this way results from the classical ergodic measures

$$
\mu_{p, q}\left(p^{\prime}, q^{\prime}\right)=\lim _{T \rightarrow \infty} \frac{1}{T} \int_{0}^{T} \mathrm{~d} t \delta\left[\left(p^{\prime}, q^{\prime}\right)-(p(t), q(t))\right]
$$

generated by a trajectory by coarse graining it both in the phase-space point $\left(p^{\prime}, q^{\prime}\right)$ and the initial point $(p, q)$.

The link between the quantum and classical probability distributions can be established using the Wigner-Weyl phase-space formalism (for a review see, e.g., $[36,37])$, which allows to assign to each projector $\hat{\varrho}=|\alpha\rangle\langle\alpha|$ a phasespace function $w_{\alpha}(p, q)$ such that the overlap of two states can be written as an integral over the product of their phase-space functions. Within this formalism, the definition (26) of the time-averaged density reads

$$
\varrho^{\mathrm{cl}}\left(\alpha, \alpha^{\prime}, t\right)=\int \mathrm{d} p \mathrm{~d} q w_{\alpha}(p, q, t) w_{\alpha^{\prime}}(p, q),
$$

exactly as in the definition of the classical density (31).

Therefore, we conclude that the quantum probability distribution $\varrho\left(\alpha, \alpha^{\prime}\right)$ is an appropriate quantity to describe quantum localization. By computing the entropy (28) of the quantum probability distribution, the degree of localization can be quantified and - by comparison with the entropy of the corresponding classical probability distribution $\varrho^{\text {cl }}\left(\alpha, \alpha^{\prime}\right)$ - quantum localization phenomena can be detected, which manifest on a time scale smaller than the classical ergodicity time scale. The latter refers to the time a classical wave packet needs to explore the available phase-space area, i.e. the time $T$ the time-averaged density (32) needs in order to converge. It is not essential to compute the Shannon entropy as a measure of localization, but one may compute any Renyi entropy (5) of the density $\varrho\left(\alpha, \alpha^{\prime}\right)$ as, e.g., $S^{(2)}=\int \mathrm{d} \mu_{\alpha^{\prime}} \varrho^{2}\left(\alpha, \alpha^{\prime}\right)$.

There is, however, a difference between the quantum and the corresponding classical entropy in the presence of discrete symmetries. The origin of the difference is that in the quantum system tunneling will occur between symmetry related phase-space regions, whereas classically such regions may belong to different invariant sets. In case of a $k$-fold symmetric structure this leads to an additional term of $\ln k$ in the quantum entropy compared to the classical one. This inherent difference can, however, be removed by 
de-symmetrizing the system, i.e. identifying symmetry related phase-space regions.

The Wigner function is in general a real but not necessarily a positive phase-space function. Therefore it cannot be unambiguously interpreted as a classical phase-space density. This problem does, however, not occur if one considers coherent states as a basis, which are the only pure states with strictly positive Wigner functions [38].

The well known coherent states $\{|\alpha\rangle=|p, q\rangle\}$ of the harmonic oscillator are eigenstates of the annihilation operator $\hat{a}=(\sigma \hat{q}-\mathrm{i} \hat{p}) / \sqrt{2 \hbar \sigma}$, i.e., $\hat{a}|\alpha\rangle=$ $\alpha|\alpha\rangle$ with $\alpha=(\sigma q-\mathrm{i} p) / \sqrt{2 \hbar \sigma}$. They can be generated from the vacuum state (defined via $\hat{a}|0\rangle=0$ ) by the displacement operator $\hat{D}(\alpha)=\exp \left[\alpha \hat{a}^{\dagger}-\alpha^{*} \hat{a}\right]$. These coherent states form an overcomplete basis of minimum uncertainty states:

$$
\mathbb{I}=\int \frac{\mathrm{d}^{2} \alpha}{\pi}|\alpha\rangle\left\langle\alpha\left|=\int \frac{\mathrm{d} p \mathrm{~d} q}{2 \pi \hbar}\right| p, q\right\rangle\langle p, q| .
$$

Their Wigner symbol is a Gaussian centered at $(p, q)$ with widths $(\Delta p)^{2}=\sigma \hbar$ and $(\Delta q)^{2}=\hbar / \sigma$; the parameter $\sigma$ is refered to as squeezing parameter. The Wigner function of coherent states is thus strictly positive and converges in the classical limit to a delta function on $(p, q)$. More general coherent states are parametrized by a complex squeezing parameter whose polar angle accounts for an additional rotation in phase-space. In $f$-dimensional systems coherent states are conveniently defined as a product of $f$ one-dimensional coherent states. For a discussion of the most general coherent states in $2 f$ dimensional phase-space see the work by Hagedorn [39]. There it is also mathematically rigorous shown that the propagation of quantum coherent states follows that of the corresponding classical phase-space distribution up to a time scale which goes to infinity in the classical limit.

Propagation of coherent states is therefore a preferable tool to study quantum/classical correspondence in Hamiltonian systems, and in particular deviations from it, which manifest themselves in localization phenomena.

With the choice of coherent states as a basis set the time-averaged probability distribution (26) becomes the time-average of the Husimi density $\varrho_{\sigma}\left(\alpha, \alpha^{\prime}, t\right)=\left|\left\langle p, q, t \mid p^{\prime}, q^{\prime}\right\rangle\right|^{2}$ of initial Gaussian wave packets. The resulting probability matrix and the entropy computed from it depend on the squeezing parameter $\sigma$ of the coherent states, although the results seem (according do our experience) not to change essentially if the parameter is varied. For- 
mally, however, one can define an entropy independent of any such parameter as the infimum over the parameter, i.e.,

$$
S(\alpha)=\inf _{\sigma}\left(-\int \frac{\mathrm{d}^{2 f} \alpha}{\pi^{f}} \varrho_{\sigma}\left(\alpha, \alpha^{\prime}\right) \ln \varrho_{\sigma}\left(\alpha, \alpha^{\prime}\right)\right) .
$$

This means that for each phase space point $\alpha$, the squeezing parameter is optimized such that the resulting time-averaged phase-space density $\varrho\left(\alpha, \alpha^{\prime}\right)$ occupies a minimum phase space volume.

\section{Quantum fluctuations in the entropy}

The second advantage of the suggested entropy concept is that the influence of inherent quantum fluctuations is reduced to order $N^{-1}$ and is therefore small in the semiclassical limit. This is easily seen by looking on the fluctuations of the matrix elements $\varrho\left(\alpha, \alpha^{\prime}\right)$ using their relation $(27)$ to the projections $p_{n, \alpha}$. If these weights are random variables, the matrix $\varrho\left(\alpha, \alpha^{\prime}\right)$ can be considered as a doubly stochastic matrix, i.e. its columns and rows both are random probability distributions. From (13), one immediately obtains as the mean value of the matrix elements

$$
\overline{\varrho\left(\alpha, \alpha^{\prime}\right)}=\frac{1}{N \nu+2}\left(\nu+2\left|\left\langle\alpha \mid \alpha^{\prime}\right\rangle\right|^{2}\right) .
$$

The overemphasis of the diagonal terms $\varrho\left(\alpha, \alpha^{\prime}\right)$ by a factor of $1+2 / \nu$ is the well-known weak localization phenomenon.

We were not able to derive the exact expression for the variance of the matrix elements $\varrho\left(\alpha, \alpha^{\prime}\right)$. Under the assumption of uncorrelated vector components $p_{\alpha, n}$, however, one easily finds

$$
\overline{\Delta \varrho^{2}\left(\alpha, \alpha^{\prime}\right)}=\frac{4}{(\nu N)^{3}}\left(\nu(\nu+1)+2 \nu\left|\left\langle\alpha \mid \alpha^{\prime}\right\rangle\right|^{2}+\left(\nu^{2}+7 \nu+12\right)\left|\left\langle\alpha \mid \alpha^{\prime}\right\rangle\right|^{4}\right) \text {. }
$$

This expression overestimates the fluctuations in $\varrho\left(\alpha, \alpha^{\prime}\right)$ even in the limit $N \rightarrow \infty$ since the error due to neglecting the correlation between different vector components $p_{\alpha, n}$ and $p_{\alpha, n^{\prime}}$ is of the same order of magnitude as the result. The scaling with $N$ is, however, obtained to the correct order which is

$$
\frac{\Delta \varrho\left(\alpha, \alpha^{\prime}\right)}{\varrho\left(\alpha, \alpha^{\prime}\right)} \sim \frac{1}{\sqrt{N}}
$$


The fluctuations in the matrix elements $\varrho\left(\alpha, \alpha^{\prime}\right)$ vanish thus in the classical limit $N \rightarrow \infty$. For almost every vector $|\alpha\rangle$ the entropy (28) will therefore be the maximum entropy minus small deviations of order $N^{-1}$ :

$$
S=\ln N\left(1-\mathcal{O}\left(N^{-1}\right)\right) .
$$

The influence of the weak localization on the entropy is thereby of the same order of magnitude as the fluctuations caused by the random fluctuations in the matrix elements $\varrho\left(\alpha, \alpha^{\prime}\right)$. Compared to the statistical properties of the entropy of an vector $|\alpha\rangle$ in the eigenbasis discussed in Sect. 3, one finds the fluctuations to be smaller by a factor of $\ln N / \sqrt{N}$, and in addition the absence of the constant symmetry related shift in the mean entropy compared to the maximum entropy.

The classical interpretation and the limit of the entropy can be best interpreted using coherent states as a basis. Suppose the classical dynamics is ergodic on a chaotic sea $A$ with volume $V$. The number of independent states which can localize on this area can be estimated to be $N \approx V / h^{f}$ according to Weyl's rule (For a more sophisticated treatment of the relation between phase-space cells and quantum projectors, see Omnes [40].). Classically, each Gaussian initially located inside the chaotic sea will fill it ergodically. The resulting classical time-averaged probability distribution (32) is therefore

$$
\varrho^{c l}\left(p, q, p^{\prime}, q^{\prime}\right)= \begin{cases}1 / V & \left(p^{\prime}, q^{\prime}\right) \in A \\ 0 & \left(p^{\prime}, q^{\prime}\right) \notin A\end{cases}
$$

with some smooth transition on the boundary of $A$ depending on $\hbar$. The classical entropy is thus

$$
S^{\mathrm{cl}}(p, q)=\ln \left(V / h^{f}\right)
$$

with some deviations of lower order in $\hbar$ due to the Gaussian smoothing of the boundary. Assuming now that the quantum evolution of the coherent state centered inside $A$ follows the ergodic classical motion sufficient long such that the wave packets behave like a completely delocalized random state, the statistical considerations above imply that the quantum entropy agrees with the classical one up to relative deviations of order $\hbar^{f}$ due to random quantum fluctuations, i.e.

$$
S(p, q)=S^{\mathrm{cl}}(p, q)+\mathcal{O}\left(\hbar^{f} \ln \hbar^{-1}\right)
$$


In contrast to the fluctuations of order $\sqrt{\hbar^{f}}$, as exposed by the entropy of coherent states in the eigenbasis, these inherent quantum fluctuations are small in the semiclassical regime. Therefore we claim that the entropy suggested here is - by comparing it with the corresponding classical entropy - an appropriate tool to detect quantum localization phenomena which manifest on a time scale smaller than the classical ergodic time scale.

\section{Illustrative Example}

In order to demonstrate the usefulness of the proposed entropy we show an example computed for a periodically driven rotor and compare this with the corresponding classical entropy as well as the quantum entropy of coherent states in the quasi-energy basis of the system. The continuously driven rotor described by the Hamiltonian

$$
H(t)=\frac{J^{2}}{2}-f \cos \phi \cos \omega t
$$

has been comprehensively studied both classically and quantum mechanically (see [7, 41, 23] and references therein). The system is also referred to as double resonance model since it shows two resonances at $J \approx \pm \omega$ which overlap according to Chirikov's criterium [42] at $f \omega^{2}=1 / 2$. Since one of the two parameters can be removed by rescaling the time, there is only one relevant classical parameter, e.g. $f$. For larger values of $f$ there is a global chaotic sea confined by adiabatic invariant curves. In addition, there are two embedded stability islands which undergo bifurcations when a parameter is changed.

For the example shown here, we have chosen $\omega=1$ and $f=1$, i.e. double the Chirikov parameter, and $\hbar=0.02$. For this value of Planck's constant, the number of states localizing on the chaotic sea is approximately $N \approx 192$; a comprehensive study of the quasi-energy states for this case has already been published in [23].

For time periodic systems the unitary Floquet operator, which is the time evolution operator $U(0, T)$ over one period $T=2 \pi / \hbar$ of the system, corresponds to the classical stroboscopic Poincaré map. The basis of the computed entropy was the time-averaged phase-space density $\varrho\left(p, q, p^{\prime}, q^{\prime}\right)$ obtained from iterating coherent states at $t=0$ under the Floquet operator. This means that instead of the time integral in (31), the sum over full periods 
$t_{k}=k T, k=0,1,2, \ldots$ has been taken. The explicit formulas for this discrete case can also be found in [14]. For the computation of the different entropies the squeezing parameter has been chosen to be $\sigma=\sqrt{1 / 2}$, i.e. adapted to the frequency of rotation around the central orbit in the center of the stability island.

In Fig. 1, first the classical entropy is shown as a contour-plot of the $3 \mathrm{D}$-graph $\{J, \phi, S(J, \phi)\}$; the distance of the contour lines is chosen to be 0.1 . Because of symmetry only the half-plane with positive momentum $J$ is shown. One recognizes a plateau of high entropy in the range $S_{\mathrm{cl}}=5.29 \pm 0.01$ (the small fluctuations are due to inaccuracy in the computation). This value is a bit larger than the value of $S=\ln (V / h)=5.22$ predicted from the area of the chaotic sea (compare (40)). This deviation is due to the Gaussian smoothing of the boundary of the sea which leads to an increase in entropy.

Outside the chaotic sea, where the dynamics is restricted to invariant curves, the entropy falls off to a lower value. The central stability island appears in the entropy plot as a valley with a minimum value close to $S=$ $1+\ln 2$, where the logarithm of 2 occurs since the island has a symmetric partner at negative momentum [14]. A further visible feature are three small dips in the entropy plot caused by tiny islands of size less than $0.03 h$ each.

The diagram of the quantum entropy (28) computed from discrete timepropagation of coherent states shown in Fig. 2 exhibits the same overall structure as the classical one in Fig. 1. The value of the entropy on the chaotic sea fluctuates around a value of $S=5.27$ which is almost identical to the classical value of $S_{\mathrm{cl}}=5.29$. The mean fluctuations of the entropy around this value are approximately $\Delta S=0.025$ such that they are not visible in the contour plot with intervals of 0.1 . Outside of the chaotic sea the quantum and the classical entropy agree as well.

One observes, however, that the tiny period-3 islands are much stronger pronounced in the quantum entropy compared to the classical entropy in Fig. 1. Here, one clearly observes a quantum localization phenomenon, which leads to an overestimate of the amount of regularity in phase-space. This effect is also slightly visible at the boarder of the large stability island. When inspecting the individual quasi-energy states of the system one finds also states which localize on the tiny period-3 island despite of their smallness. The entropy plot in Fig. 2 , however, detects this localization in a quantitative way and without the need of inspecting all individual states. 
In the Fig. 3, we show the quantum entropy (24) of the coherent states in the quasi-energy states of the system. These states are the eigenstates of the Floquet operator, i.e. $U(0, T)|n\rangle=\mathrm{e}^{\mathrm{i} \varepsilon_{n} T / \hbar}|n\rangle$, and play in time-periodic systems the role Hamiltonion eigenstates have in time-independent systems. When comparing this entropy with that in Fig. 2, one immediately notifies the dominance of strong fluctuations, which are on the average approximately $\Delta S=0.085$ on the chaotic sea. A further difference to the entropy of the time-averaged probability densities is the smaller mean value of the entropy over the chaotic sea which is $\bar{S}=4.80$. This deviation is is close to deviation of $c_{2}=1-C=0.422$ predicted for random unitary vectors (compare (19)).

The localization on the period-3 islands is also visible in this entropy plot, but it is not clear whether this is the only statistical significant localization phenomenon in this system. One may notify other structures which might be due to some localization, but their effect on the entropy is of the same order of magnitude as the inherent statistical fluctuations due to the quantum fluctuations in the projections $|\langle J, \phi \mid n\rangle|^{2}$. This leads for instance also to the lowered entropy on the axis $\{J=0\}$. The quasi-energy states decompose in two classes with even and odd parity, respectively $[41,23]$. In the momentum representation the odd states are all equal zero at $J=0$ and therefore their overlap with coherent states located on this line will in general be smaller than that of the even states. As a result, their entropy is smaller than the average entropy on the chaotic sea. This observation is, however, not related to any dynamical localization. An advantage of the entropy shown in Fig. 2 is that these effects do not show up but instead the localization due to a quantum suppression of the classical ergodic spreading of wave packets is clearly exposed without superposition of other effects or large quantum fluctuations.

\section{Conclusion}

In this paper, we have presented a novel entropy concept measuring localization in dynamical systems. Based on time-averaged probability densities, this concept is suitable to describe the statistical relaxation of time propagated wave packets. One of the main advantages of the novel concept is that is has a classical counterpart. By comparing the quantum entropy with the corresponding classical one, localization phenomena which manifest themself 
in suppressing the classical spreading of a wave packet on a time scale smaller than the classical ergodicity time can be detected and quantified.

An interesting characteristic of the classical counterpart of the entropy is that its scaling behavior with $\hbar^{-1 / 2}$ is given by the dimension of the underlying ergodic sets of the classical dynamics. A map $(p, q) \rightarrow S(p, q)$ obtained from a quantum computation provides therefore a global quantum phase space image showing the quantum correspondence of the different classical invariant phase space sets.

Although coherent states are a preferable tool to study the transition from classical to quantum dynamics, the suggested entropy is defined with respect to any 'natural basis' set whose dynamics one wants to study in view of localization.

A further essential advantage of the novel entropy concept is that the influence of inherent quantum fluctuations on the entropy is reduced to an order of $\hbar^{f}$. Therefore the novel concept offers the possibility to quantify quantum localization in a statistical significant way, and we hope that the concept will in the be applied in future studies of localization in dynamical systems.

\section{Acknowledgement}

We thank W. Rühl for pointing out the valuable relation (18) between average entropies and beta functions.

\section{References}

[1] O. Bohigas, in Proc. 1989 Les Houches School on Chaos and Quantum Physics, edited by M.-J. Giannoni, A. Voros, and J. Zinn-Justin (NorthHolland, Amsterdam, 1989).

[2] T. A. Brody et al., Rev. Mod. Phys. 53, 385 (1981).

[3] M. L. Mehta, Random Matrices (Academic Press, Boston, 1991).

[4] S. W. McDonald and A. N. Kaufman, Phys. Rev. A 37, 3067 (1988).

[5] M. Kuś, J. Mostowski, and F. Haake, J. Phys. A 21, L1073 (1988). 
[6] R. Aurich and F. Steiner, Physica D 64, 185 (1993).

[7] N. Moiseyev, H. J. Korsch, and B. Mirbach, Z. Phys. D 29, 125 (1994).

[8] S. Fishman, D. R. Grempel, and R. E. Prange, Phys. Rev. Lett. 49, 509 (1982).

[9] D. R. Grempel, R. E. Prange, and S. Fishman, Phys. Rev. A 29, 1639 (1984).

[10] G. Casati, I. Guaneri, F. Israelev, and R. Scharf, Phys. Rev. Lett. 64, 5 (1990).

[11] D. Wintgen, K. Richter, and G. Tanner, Chaos 2, 19 (1992).

[12] F. Bensch, H. J. Korsch, B. Mirbach, and N. Ben-Tal, J. Phys. A 25, 6761 (1992).

[13] T. Geisel, G. Radons, and J. Rubner, Phys. Rev. Lett. 57, 2883 (1986).

[14] B. Mirbach and H. J. Korsch, Phys. Rev. Lett. 75, 362 (1995).

[15] G. Casati, B. Chirikov, I. Guarneri, and F. M. Izrailev, Phys. Rev. E 48, R1613 (1993).

[16] E. Heller, Phys. Rev. A 35, 1360 (1987).

[17] T. Dittrich and U. Smilansky, Nonlinearity 4, 59 (1991).

[18] A. Wehrl, Rep. Mat. Phys. 30, 119 (1991).

[19] A. Wehrl, Rev. Mod. Phys. 50, 221 (1978).

[20] A. Anderson and J. J.Halliwell, Phys. Rev. D 48, 2753 (1993).

[21] V. Bužek, C. H. Keitel, and P. L. Knight, Phys. Rev. A 51, 2575 (1995).

[22] V. Bužek, C. H. Keitel, and P. L. Knight, Phys. Rev. A 51, 2594 (1995).

[23] T. Gorin, H. J. Korsch, and B. Mirbach, Chem. Phys. 217, 147 (1997), special Issue on 'Dynamics of Driven Quantum Systems'.

[24] S. D. Frischat and E. Doron, J. Phys. A 30, 3613 (1997). 
[25] W. K. Wootters, Found. Phys. 20, 1365 (1990).

[26] K. Życzkowski, J. Phys. A 23, 4427 (1990).

[27] T. Zimmermann, H. Köppel, and L. S. Cederbaum, J. Chem. Phys. 91, 3934 (1989).

[28] F. Haake, Quantum Signatures of Chaos (Springer, Berlin, Heidelberg, New York, 1992).

[29] K. R. W. Jones, J. Phys. A 23, L1247 (1990).

[30] R. Abraham and J. E. Marsden, Foundations of Mechanics (Benjamin, Reading, 1978).

[31] F. M. Israelev, J. Phys. A 22, 865 (1989).

[32] M. Abramowitz and I. A. Stegun, Handbook of Mathematical Functions (Dover Publications, New York, 1970).

[33] E. Thiele and J. Stone, J. Chem. Phys. 80, 5187 (1984).

[34] K. S. L. Nordholm and S. A. Rice, J. Chem. Phys. 61, 203 (1974); 61, 768 (1974).

[35] J. A. Núñez, P. M. Cincotta, and F. C. Wachlin, in Celestrial Mechanics and Dynamical Astronomy 64 (1996) (Kluwer Academic Publishers, Dordrecht, 1996), p. 43, conference: Chaos in Gravitational N-Body Systems, La Plata, 1995.

[36] S. R. DeGroot and L. G. Suttorp, Foundations of Electrodynamics (North-Holland, Amsterdam, 1972).

[37] M. Hillery, R. F. O‘Connel, M. O. Scully, and E. P. Wigner, Phys. Rep. 106, 121 (1984).

[38] N. Lütkenhaus and S. M. Barnett, Phys. Rev. A 51, 3340 (1995).

[39] G. A. Hagedorn, CMP 71, 77 (1980).

[40] R. Omnes, J. Stat. Phys. 57, 357 (1989). 
[41] V. Averbuckh, N. Moiseyev, B. Mirbach, and H. J. Korsch, Z. Phys. D 35, 247 (1995).

[42] B. V. Chirikov, Phys. Rep. 52, 263 (1979). 


\section{Figure Captions}

Fig. 1: Classical entropy computed from the time-averaged phase-space probability distributions of iterated classical Gaussian densitities using a squeezing parameter of $\sigma=\sqrt{1 / 2}$. The distance of the contour lines is 0.1 . One recognizes a plateau of high entropy with an embedded stability island and three tiny island of area less than $0.01 h$.

Fig. 2: Quantum entropy computed from the time-averaged Husimi density. The comparison with Fig. 1 shows a quantum localization on the tiny regular islands.

Fig. 3: Entropy of the coherent states expanded in the quasi-energy states of the system, i.e. $S(J, \phi)=-\sum_{n}|\langle J, \phi \mid n\rangle|^{2} \ln |\langle J, \phi \mid n\rangle|^{2}$. The plot is dominated by strong fluctuations. 
Figure 1:

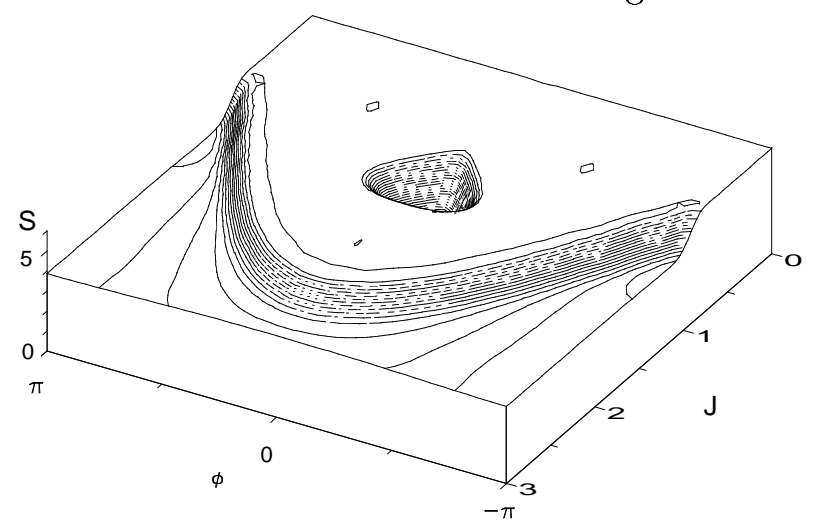

Figure 2:

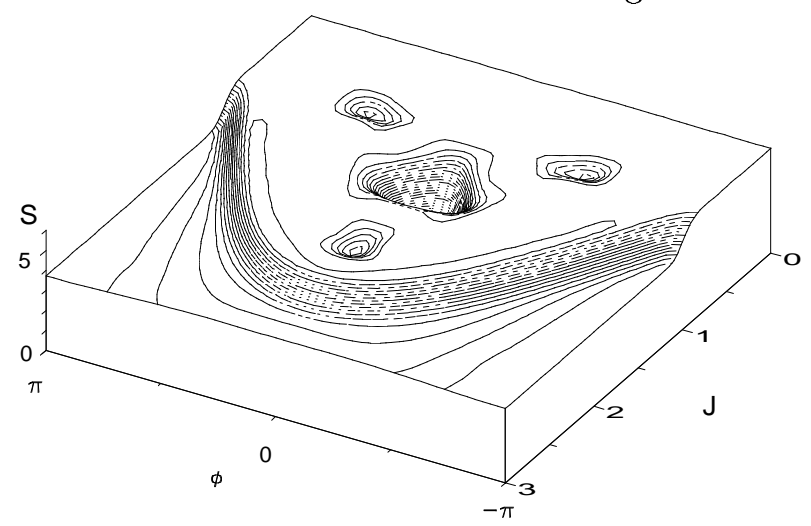

Figure 3:

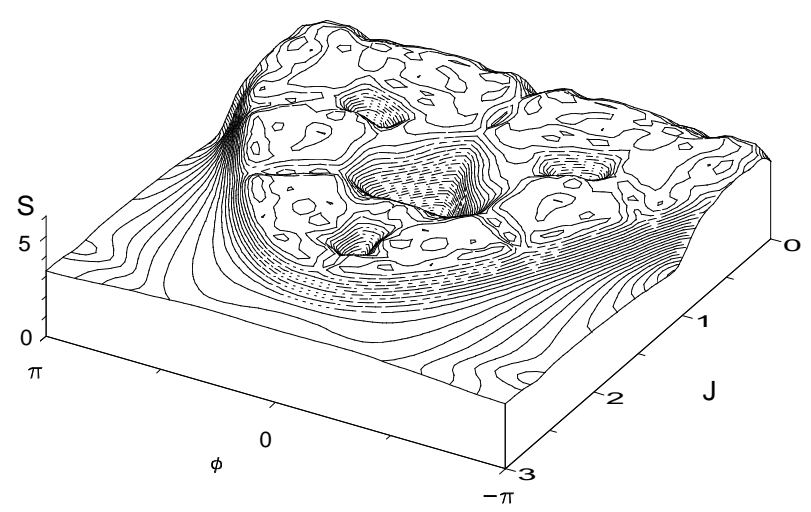

